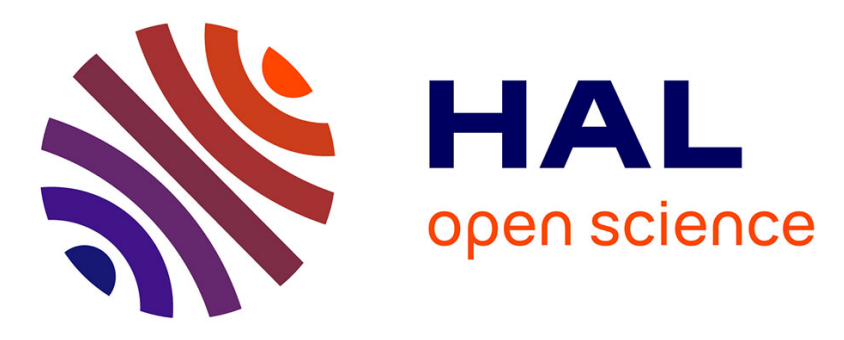

\title{
Multiple Moving Targets Tracking based on Kernel Localization and Group Trackers for Envisioned Functional Microwave Brain Imaging Applications
}

\author{
Mohammad Ojaroudi, Stéphane Bila
}

\section{- To cite this version:}

Mohammad Ojaroudi, Stéphane Bila. Multiple Moving Targets Tracking based on Kernel Localization and Group Trackers for Envisioned Functional Microwave Brain Imaging Applications. 2020 14th European Conference on Antennas and Propagation (EuCAP), Mar 2020, Copenhagen, Denmark. pp.1-5, 10.23919/EuCAP48036.2020.9135553 . hal-03047716

\section{HAL Id: hal-03047716 \\ https://hal-unilim.archives-ouvertes.fr/hal-03047716}

Submitted on 8 Dec 2020

HAL is a multi-disciplinary open access archive for the deposit and dissemination of scientific research documents, whether they are published or not. The documents may come from teaching and research institutions in France or abroad, or from public or private research centers.
L'archive ouverte pluridisciplinaire HAL, est destinée au dépôt et à la diffusion de documents scientifiques de niveau recherche, publiés ou non, émanant des établissements d'enseignement et de recherche français ou étrangers, des laboratoires publics ou privés. 


\title{
Multiple Moving Targets Tracking based on Kernel Localization and Group Trackers for Envisioned Functional Microwave Brain Imaging Applications
}

\author{
Mohammad Ojaroudi ${ }^{1}$, and Stéphane Bila ${ }^{1}$ \\ ${ }^{1}$ XLIM, UMR no7252, University of Limoges, 123 Av. Albert Thomas, 87060 Limoges, France \\ e-mail: mohammad.ojaroudi@xlim.fr
}

\begin{abstract}
This paper presents a new concept of multiple target tracking using hierarchical trackers based on kernel localization for envisioned functional microwave brain imaging application. For this purpose, the performance of brain activated regions tracking using fMRI video is improved. In the first stage, all of the moving regions in the fMRI video frame are detected. Then, by using the group tracking, histogram and distance corresponding to the moving-targets in the previous frame, the directions of moving-regions are determined. After determining the exact number of moving objects and tracking them, one by one, the direction of each vector is extracted. In addition, due to the kernel labelling, the proposed method has capability of separation and merging by group tracking in conditions of motion paths interfere with each other. The simulated results validate the effectiveness of the proposed methods for precisely tracking of the activated regions.
\end{abstract}

Index Terms-functional magnetic resonance imaging (fMRI), functional microwave brain imaging system, multiple moving-targets tracking, histogram based kernel detection, group tracking.

\section{INTRODUCTION}

Among neuro imaging techniques, functional magnetic resonance imaging (fMRI) is currently the most widely used as it can provide excellent detailed anatomical and physiological information related to pathologies such as brain tumours, Alzheimer's disease, and epilepsy [1]. In physiological studies such as functional magnetic resonance imaging (fMRI), the precise location of the activated brain regions must be known to make the data meaningful [2]. Due to their mechanical complexity and excessively large size, magnetic resonance imaging is not practical for long term continuous monitoring. In addition, metallic objects are prohibited, thereby excluding patients with a surgical prosthesis or pace maker [3-4]. Recently microwave imaging system has been considered as a new modality for brain imaging system. An array based UWB microwave imaging system has great potential to provide the means of noncontact long-term continuous monitoring of cancerous tissues, brain stroke tomography, and even functional neuro imaging not possible using other existing techniques [5-6].

Multiple moving targets tracking is fundamental to many higher-level applications such as behavioural analysis, loitering detection, crowd analysis, cell identification, visual-based animal tracking and people counting systems
[7-10]. Multiple-moving-targets trackers provide the user with the path of each tracked moving-targets in the system. The level of difficulty is several times greater than single moving-targets tracking because of track swapping issues, complex data association, merging, splitting and condensed fields (making it difficult to update tracker appearance information). Examples of common methods for multiple moving targets tracking are the particle filter [7], joint probabilistic data association filter [8], hidden Markovbased filter [9], probability hypothesis density filter [10] and multiple hypothesis tracker [11]. A good review of visionbased moving-targets tracking comprised of various detection schemes appears in [12]. Most appearance-based detection schemes are implemented by using the mean-shift or Kernel-based tracker [13]. Such methods gather statistical properties of the detected moving-targets, particularly colour histograms, and predict the moving-targets' next location by matching the properties in the following frames. These approaches require frequent update of appearance information and become less accurate as the scene becomes more crowded. However, such a system is robust to complex scene variation, especially for single movingtargets tracking [14].

In this paper, we present a novel robust hierarchical multiple hypotheses tracker (MHT) for multiple moving targets tracking in a sample of fMRI video. In fMRI video analytics, the region of the tracked moving-targets can vary significantly, creating a challenge in getting the right bounding box since it is rare to detect the entire movingtargets perfectly. Mergers and fragments issues mean observation association needed to update the tracks. In this context, considering several hypotheses instead of using a single soft or hard-based decision is led preferable trackers. Since we only accept first level input with correspondence, the velocity value is more stable, which is important to distinguish occlusions, mergers and brief intersection cases. The first level detect small tracklets using a two-threshold strategy based on affinity of the detections. The second level uses maximum posteriori formulation to find the best association based on appearance and motion. The highest level determines whether the tracklets are entering, leaving or being occluded by other tracks. Simulated results show that the proposed methods could be a good candidate for multiple target tracking for brain activities monitoring applications. 


\section{FUnCTIONAL MAGNETIC RESONANCE IMAGING} VERSus Functional MicrowaVE BRAIN IMAGING SYSTEM

\section{A. Functional Magnetic Resonance Imaging (fMRI)}

In typical fMRI experiment, the goal is to map patterns of neuronal activation in the subject's brain while performing specific tasks. Instead of measuring the neuronal activity in fMRI, the blood-oxygen-level-dependent (BOLD) effect in response to activation is determined. In fact, BOLD is sensitive to the concentration change of deoxygenated hemoglobin, which in turn is dependent on cerebral blood flow $(\mathrm{CBF})$, cerebral blood volume $(\mathrm{CBV})$, and cerebral metabolic rate of oxygen (CMRO2) [15]. The main goal for interpreting fMRI data is to figure out the underlying link between neuronal activities and the brain's hemodynamic response. In the illustration of Fig. 1, a flickering checkerboard stimulus triggers excited neuronal activity in the visual cortex [15]. This is going along by increased blood flow, blood volume, and oxygen metabolism, and these physiologic changes merge to change the local deoxyhemoglobin, which in turn changes the local MR signal.

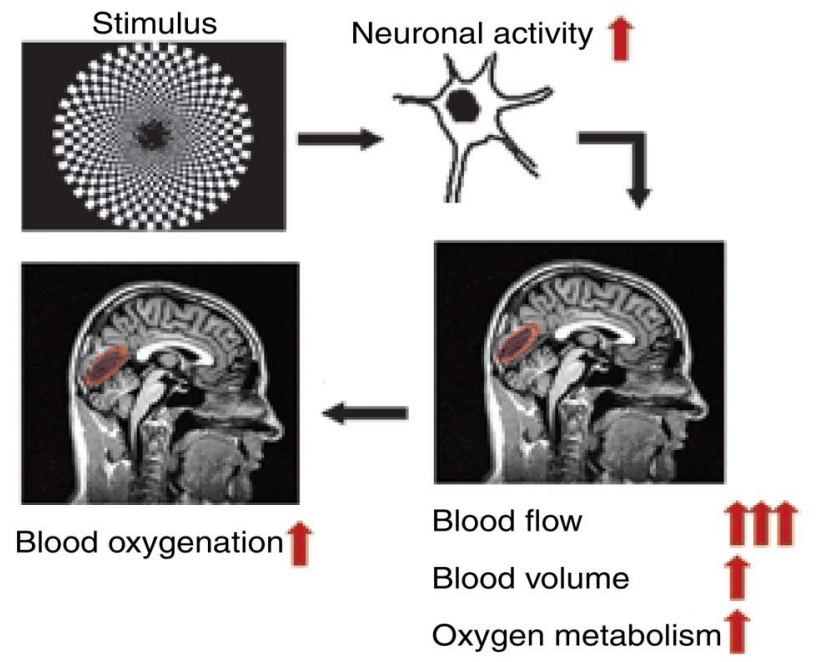

Fig. 1. Block design experiment to measure the local BOLD signal changes in a subject's brain during an fMRI experiment [15].

Block designs experiment is used as geographic mapping of the fMRI. In this perspective for one of the experimental paradigms, stimuli are introduced in alternating short runs ("blocks") of several seconds' interval, and the MRI signals are then compared for the two types of blocks. For instance in visual-stimulation task to centralize primary visual areas, a subject might view a bright flickering checkerboard for 20 seconds, pursued by a dark screen for 20 seconds, with these blocks repeated several times; eight pairs of blocks, would require a total recording that lasts under 6 minutes. During that time, images may be recorded for several different parallel slices (typically 10-20), such that each slice is imaged about every 2 seconds. In this case, 80 images would be acquired for each slice for both conditions (stimulus ON and OFF) [16]. Those volume elements (voxels) within the brain that are affected by the stimulus prepare an interval of data points. Because of the BOLD effect, the signal alternates in strength in synchrony with the stimulation (Fig. 2). By detection of which voxels show this alternating pattern, the visual cortex can be identified.

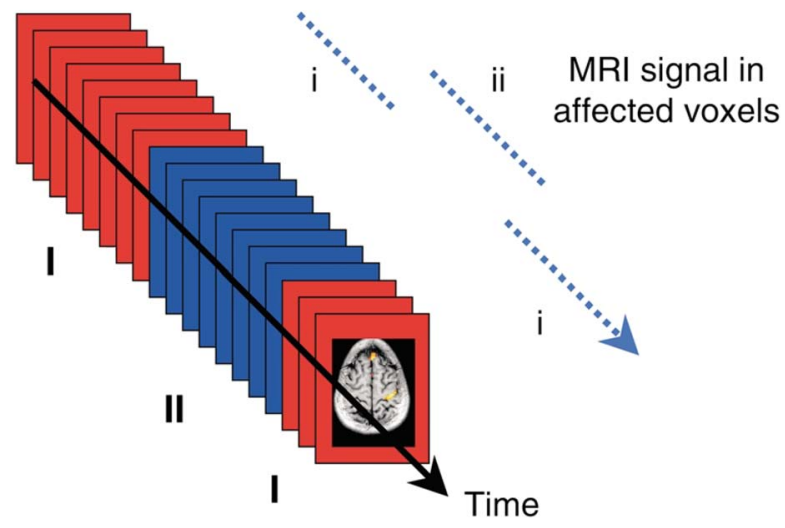

Fig. 2. In a block design experiment, sequences of images are integrated in contrasting conditions (I and II). After integration, those voxels whose signals change in synchrony (i to ii) with the stimulus can be identified [16].

\section{B. Functional Microwave Baain Imaging System}

In the envisioned functional microwave brain imaging system perspective, the underlying hypothesis is that, if we can detect local changes in blood volume inside the brain precisely enough, we can infer which parts of the brain are active when performing various tasks such as moving various muscles, making decisions, experiencing emotions and others [5]. The proposed approach involves illuminating brain with an ultra-wideband pulse from an antenna location, then synthetically focusing reflections from the biological tissue as shown in Fig. 3 (a). In addition, Fig. 3 (b) shows generic algorithm/architecture co-design based on knowledge extraction and information fusion for the proposed functional microwave imaging system. As shown in Fig. 3 (b), in order to create cognitive scanning capability in system level design we are faced to with there types of dynamic flow such as data, control and information.

In information-based scanning, we face with random spatial variations in the activities of the brain regions, a randomness which is due to the diversity of the regions that must be intelligently delimited and observed. This means that the movement patterns of the activated regions and the arrangement of these movements are random, i.e., hierarchical activation or simultaneous casting in the next slides varies randomly. In this point of view by relying on cognitive scanning techniques and the proposed reconfigurable structure, it aims at developing an innovative desiccation making scheme suitable to UWB functional microwave imaging system. In order to extract anatomical knowledge and physiological information fusion of the generated 3D images (from the reflected signal sequences), it is necessary to make perception action loop in cognitive algorithms, which will be controlled by 
switching matrix in the architecture section. The proposed cognitive scanning program prepares code division in order to provide a set of smart pulse shaping scheme and array configuration based on self-organizational techniques.

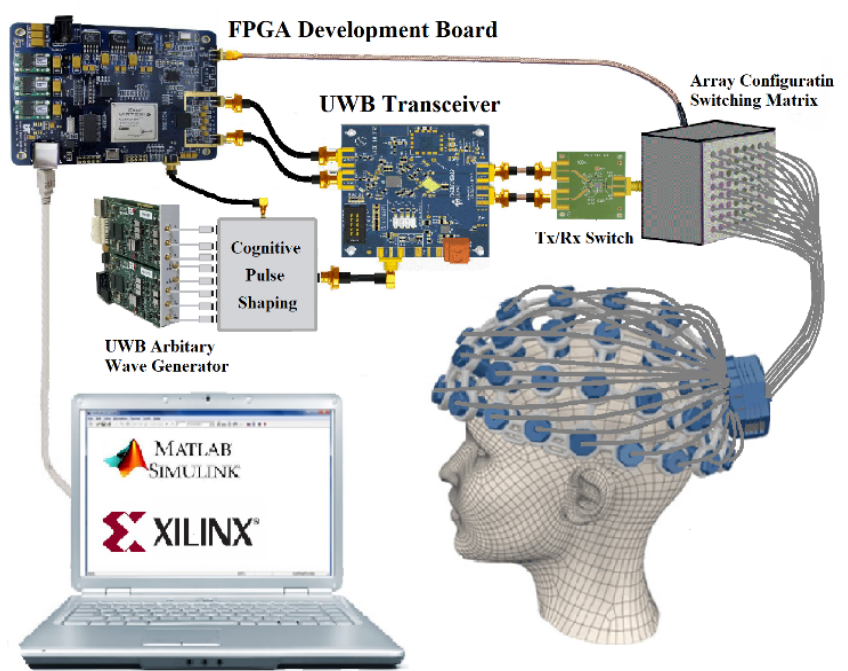

(a)

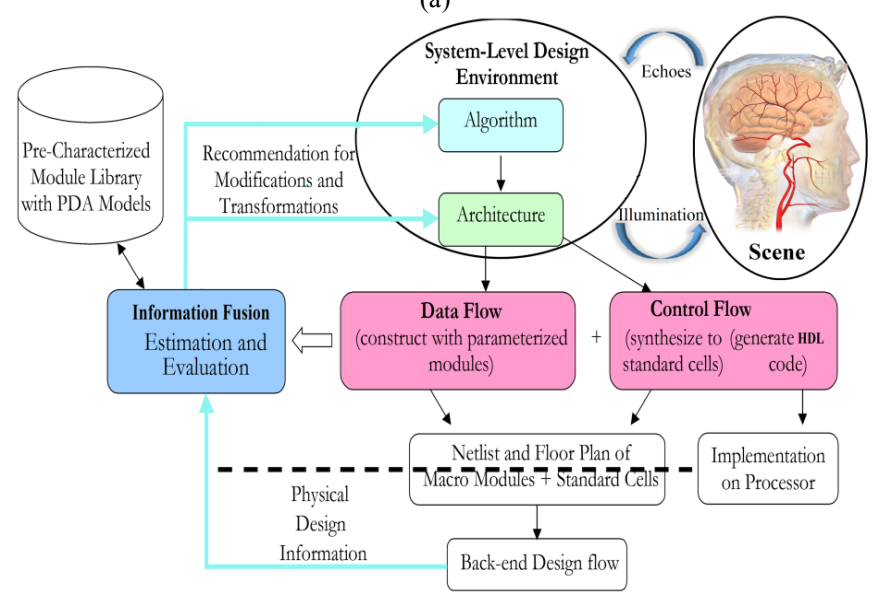

(b)

Fig. 3. (a) Hardware backbone of the proposed functional microwave brain imaging framework, and (b) Schematic illustration of the envisioned algorithm/architecture co-design based on knowledge extraction and information fusion for functional microwave imaging system [5].

Fig. 4 compares the proposed functional imaging system with the conventional differential imaging paradigm. In this point of view, extracted information adds new anatomical information in the library of the knowledgegrowing system (KGS) to estimate future locations and moves of each target of interest (TOI). In addition, according to neurological outcomes, for the normal functioning of the brain, the activations zones are known [5]. That means that we are able to create a library to compare various states and estimate the next move. The closed-loop cognitive scanning can be considered as a valuable real alternative to differential imaging, which an is faster alternative, which is faster, and more information can be extracted. In contrast to open-loop differential imaging, closed-loop cognitive functional imaging is modulated in real-time through the feedback from recordings of backscatters to intelligent illumination [5]. To achieve this, according to dynamic changes in brain activities, the transmitted waveforms, and thus the activated sub-arrays, phased arrays and focusing arrays structures will be cognitively modified and continuously adapted to the changing targets. a)

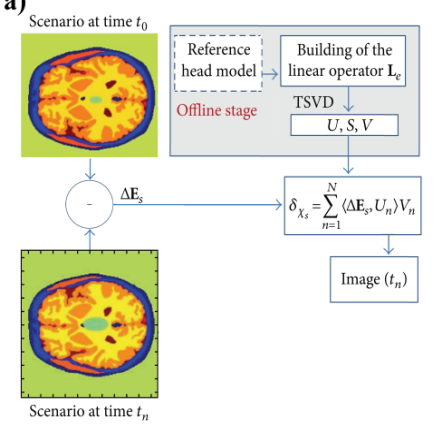

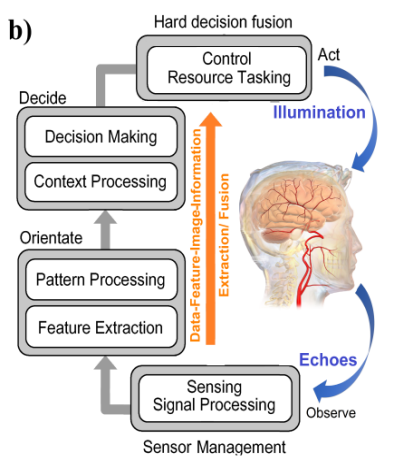

Fig. 4. (a) Conventional open-loop differential imaging for dynamic imaging [24], and (b) A new frontier in closed-loop imaging based on the proposed cognitive imaging process.

\section{THE Proposed HiERARChICAL MUltiple HYPOTHESES TRACKER (MHT)}

In order to propose a novel approach of multiple moving targets tracking in fMRI video, the proposed hierarchical multiple hypothesis tracking algorithm is shown in Fig. 5. By implementing a multiple-hypothesis approach, the tracker can combine all possible fragments to get the best observations. For the first stage, it uses K-best hypotheses incorporating adjacency information. For the second stage, the algorithm tries to find the best hypothesis by considering the merge and split case. The alignment of the measurement box dependents on whether its size is bigger than the predicted states. If the measurement box is bigger, the center point remains the same while if it is smaller, the center point of the predicted measurement will be shifted inside the observation box using the minimum distance. Also, by fixing the moving-targets' size, the association will tend to reject big-box measurements, which will lead to track deletion after several updates without correspondence [13]. In this algorithm, the MHT implementation depends on histogram intersections for calculating the quality of the hypothesis. It models a histogram similarity function in HSV color space where the best hypothesis is obtained by merging fragments, resulting in the highest histogram intersection value.

Both stages' inputs act independently, where the quality of all hypotheses are measured based on Gaussian modeling of centroid's position, velocity, orientation and size of the blobs [13]. The outputs of the first level are then fed into the second level MHT with a portion of a big foreground blob. The first level is mainly to obtain stable velocity values and to filter out false track. The big background blob is the area that contains more than one moving-targets. The blob needs to be partitioned based on predicted state. 


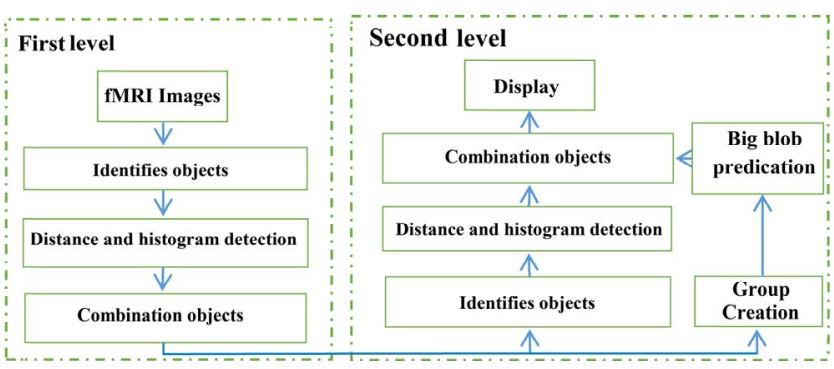

Fig. 5. Flow chart of the proposed multiple-target tracking method.

The second level tracker only accept first level outputs, which have at least one correspondence at any particular scan. The reasons are to reduce noisy observations and a good velocity estimates can only be obtained if a track has correspondence. In this step, the best combination of the observations is identified by considering merge, split, fragments and occlusion methods. Before the association is performed, a collision detector based on the overlapping region among the predicted states is run. We can assign more weight to movement flow detection relative to foreground segmentation by knowing which track has a higher possibility of being occluded. Clustered movement flow alone can distinguish occlusion but not a merge case. With advances in detection accuracy, the real activated brain regions are presented to a tracking processing layer as a set of multiple reflection points. Those detection points form a group of correlated measurements with range, angle, and angular velocity. Therefore, we seek a tracker capable of working with multiple target groups in multiple reflection point's scenario. The schematic of the utilized group tracking approach is illustrated in Fig. 6.

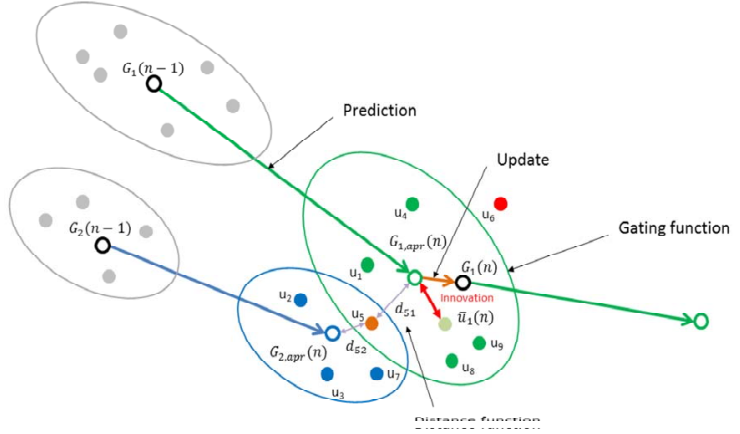

Fig. 6. The group tracking approach for merging the neighboring regions [17].

In addition, the group tracking approach is considered for merging the neighboring regions. For this purpose, first, the kernel-based prediction process is used to estimate tracking group centroid for time $\mathrm{n}$ based on estimated state covariance matrices at time $\mathrm{n}-1$. It is done by computing a-priori state and covariance estimations for each trackable object. At this step, we also compute vector estimations. Then, existence of one or more tracks and the associated predicted state vector is assumed. For each given track, a gate is formed by the predicted centroid. Then the group residual covariance matrix is used to build an ellipsoid in 3D space based on tracking group centroid. The ellipsoid represents a gating function by computing normalized distance function as a cost function at each track. The assignment process minimizes the cost function, assigning one measurement at a time to the closest track. This creates a set of measurements associated with each track [17].

\section{RESULTS AND DISCUSSIONS}

In order to illustrate the proposed method performance, we used brain's conventional fMRI video with a time varying regions. Fig. 7 shows only six imaging-shots samples in first five seconds of the selected fMRI video [18]. This image demonstrates multiple-moving regions, which is marked by the changing of the color in each region. It can be found that in all pictures, targets regions appear very clearly; which indicates that brain activity during fMRI tasks. It is also easy to find contrast of target and activated regions in Fig. 7 is higher than that other deactivated regions.

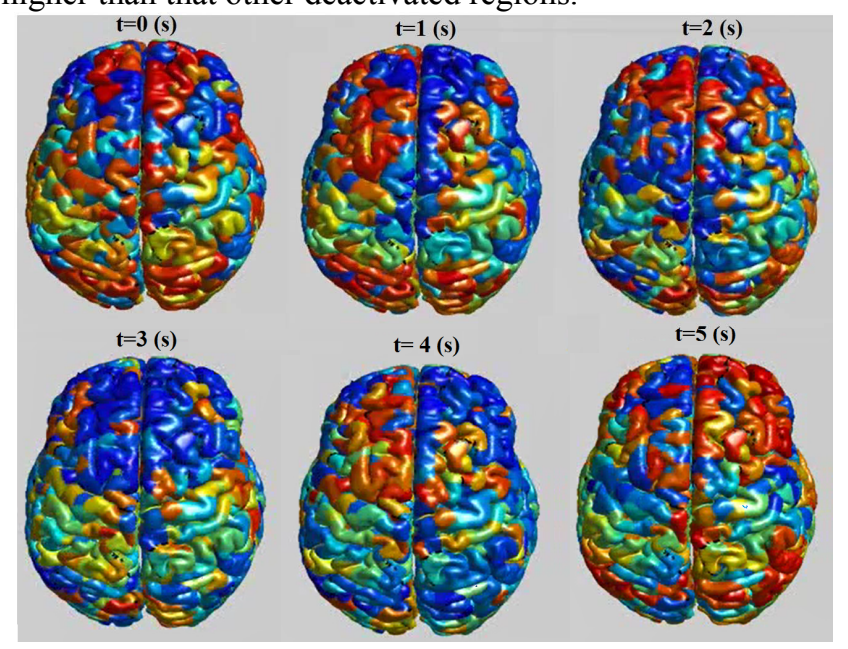

Fig. 7. Six imaging-shots samples in first five seconds of a conventional fMRI video

Fig. 8 presented the complete identification of movingtargets by selecting a frame of fMRI video to remove foreground and extracting background uses optical flow compared to three previous frame as shown in Fig.8 (a). In addition, Fig. 8 (b) shows removing spot and grain noise to restoration of the extracted background. The estimated areas of the bubbles obtained are shown in Fig. 8 (b). The chosen frames contain multiple moving-targets in various conditions and the tracking performance during merging, collision, occlusion, fragments, move-stop-move and split problems has been shown.

In Fig. 9, distance and histogram of each bubble are calculated from the previous frame to determine the direction of each bubbles. The bubbles that have less distance from each other are combined to create a new bubble that shown in Fig. 9 (b). In the second stage after grouping, the larger distance is considered to separate the group's kernels. Fig. 9 (c) shows the final merged groups which are identified as activated regions. This process is highly dependent on the size and speed of the tracked moving-targets. If the average flow velocity be reduced, a 
smaller tracked moving-target will require a lower threshold, [19]. Shadow removal only is applied to the videos that contain moving-targets, which are relatively large compared to the frame size. For small moving-targets, false alarm is preferable to miss detection [20].

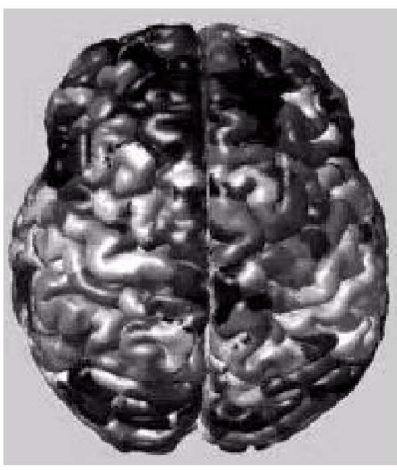

(a)

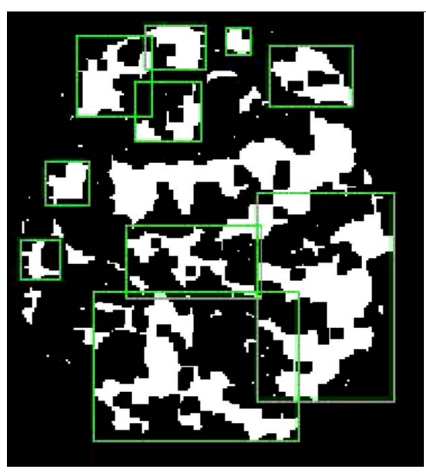

(b)
Fig. 8. Tracking and combination procedures at the first stage of the algorithm (a) The original image, (b) Background extracted and identified objects.

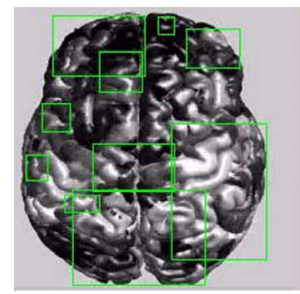

(a)

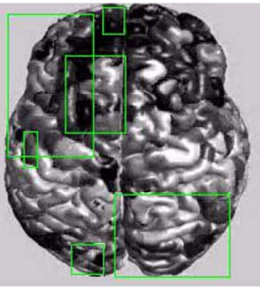

(b)

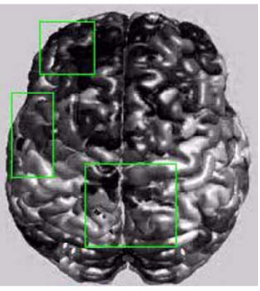

(c)
Fig. 9. Tracking procedures of second stage of the algorithm, (a) identified objects, (b) kernel detection based on distance and histogram detection, and (c) the group tracking approach for merging the neighboring regions.

\section{CONCLUSION}

In this paper, the utilization of the multiple moving targets tracker for detection activated baring regions during brain monitoring for using in functional microwave imaging system is presented. The main objective is making possible multiple target tracking in fMRI videos as a practical tool in the envisioned microwave imaging paradigm. To demonstrate this point, a novel robust hierarchical multiple hypotheses tracker (MHT) has been applied to the selected fMRI video from brain activation. In the first stage, all of the moving regions in the fMRI video frame are detected. Secondly, by using the histogram and distance corresponding to the moving-targets in the previous frame, the directions of moving-targets are detected and determined. In addition, the group tracking is used to address the activated brain regions.

\section{REFERENCES}

[1] J. Beutel, L. Harold, and L. Richard, "Handbook of medical imaging." Vol. 1. Spie Press, 2000.

[2] K. Doi, "Computer-aided diagnosis in medical imaging: historical review, current status and future potential." Computerized medical imaging and graphics, 31(4-5), pp.198-211. 2007.

[3] M. Guardiola, L. Jofre, F. Gedda, S. Capdevila, J. Romeu, and S. Blanch, "3D arrayed microwave tomographic system for medical imaging." Wireless Information Technology and Systems (ICWITS), 2010 IEEE International Conference on, pp. 1-4, August 2010.

[4] J. R. Dalenberg, H. R. Hoogeveen, and M. M. Lorist, "Physiological Measurements: EEG and fMRI." In Methods in Consumer Research, Volume 2, pp. 253-277, 2018.

[5] M. Ojaroudi, S. Bila and M. Salimi, "A Novel Approach of Brain Tumor Detection using Miniaturized High-Fidelity UWB Slot Antenna Array," 2019 13th European Conference on Antennas and Propagation (EuCAP), Krakow, Poland, pp. 1-5, 2019.

[6] M. Ojaroudi, S. Bila, P. Leveque and P. Carré, "Functional Microwave Imagining System based on Cognitive Scanning for Brain Activities Monitoring: A Feasibility Study," 13th European Conference on Antennas and Propagation (EuCAP), Krakow, Poland, pp. 1-5, 2019.

[7] Z. Khan, T. Balch, and F. Dellaert, "An MCMCbased particle filter for tracking multiple interacting targets." European Conference on Computer Vision. In T. Pajdla \& J. Matas (Eds.). Lecture Notes in Computer Science, Springer, Vol. 3024, pp. 279-290, 2004.

[8] T. E. Fortmann, Y. Bar-Shalom, and M. Scheffe, "Sonar tracking of multiple targets using joint probabilistic data association." IEEE Journal of Oceanic Engineering, 8(3), pp. 173-184, 1983.

[9] P. Natarajan, and R. Nevatia, "Real-time tracking and recognition of human actions." In IEEE workshop on motion and video computing, pp. 1-8, 2008.

[10] N. T Pham, W. Huang, and S. H. Ong, "Tracking multiple objects using probability hypothesis density filter and color measurements." IEEE international conference on multimedia, pp. 1511-1514, 2007.

[11] I. J. Cox, and S. L. Hingorani, "An efficient implementation of Reid's multiple hypothesis tracking algorithm and its evaluation for the purpose of visual tracking." IEEE Transactions on Pattern Analysis and Machine Intelligence, 18(2), 138-150, 1996.

[12] A. Yilmaz, O. Javed, and M. Shah, "Object tracking: A survey." ACM Computing Surveys, 38, 2006.

[13] A. Torabi, and F.A. Bilodeau, "A multiple hypothesis tracking method with fragmentation handling." In Canadian conference on computer and robot vision, pp. 8-15, 2009.

[14] I. Leichter, M. Lindenbaum, and E. Rivlin, "Mean shift tracking with multiple reference color histograms." Computer Vision and Image Understanding, 114, 400-408, 2009.

[15] K. Uludag, J. D. Dubowitz, and R. B. Buxton. "Basic principles of functional MRI." Clinical MRI. Elsevier, San Diego 249-287, 2005.

[16] J. C. Gore, "Principles and practice of functional MRI of the human brain." The Journal of clinical investigation 112, no. 1, pp. 4-9, 2003.

[17] M. Livshitz, "Tracking radar targets with multiple reflection points.", Texas Instruments, Incorporated 20450 Century Boulevard Germantown, MD 20874, USA- 2018.

[18] Educational talk form ISMRM 2018 in Paris: https://www.ismrm.org.

[19] A. N. Mohamed,. "A Novice Guide towards Human Motion Analysis and Understanding." arXiv preprint arXiv:1509.01074, 2015.

[20] T. Xingtian, and H. Wu. "A Comparison of the Sequential Quadratic Programing Algorithm and Extended Kalman Filter Method in the Magnetic Particle Tracking Reconstruction." In AIAA Scitech, Forum, p. 0272. 2019. 